\title{
Current status of JUNO Top Tracker
}

\author{
Q. Huang ${ }^{* a}$ and J. P. A. M. de André ${ }^{b}$ on behalf of the JUNO Collaboration \\ ${ }^{a}$ LLR, IN2P3/CNRS, Palaiseau, France \\ ${ }^{b} I P H C$, IN2P3/CNRS, Strasbourg, France \\ E-mail: qinhua.huang@llr.in2p3.fr, jpandrediphc.cnrs.fr
}

\begin{abstract}
The JUNO experiment is a multi-purpose antineutrino oscillation experiment with the main objective of determining the neutrino mass hierarchy with a sensitivity better than $3 \sigma$, which requires JUNO to have an energy resolution better than $3 \%$ at $1 \mathrm{MeV}$. The JUNO Central Detector, a $20 \mathrm{kt}$ liquid scintillator detector, will be built with high PMT photocathode coverage and good transparency for this purpose.

Despite the $700 \mathrm{~m}$ overburden, the atmospheric muon-induced background is still estimated to be non negligible compared to the expected signal for the neutrino mass hierarchy determination. A veto system was designed for muon detection to tag and to suppress this background. It consists of two subsystems: the Top Tracker and the Water Cherenkov Detector. The Top Tracker is a 3 -layer muon tracker covering about $60 \%$ of the top surface above the JUNO water pool and will provide precise tracking for atmospheric muons. These well reconstructed muons are essential in the veto strategy for rejecting cosmogenic isotope background. Combining the muon information from the Top Tracker and the Water Cherenkov Detector, most of the atmospheric muon-induced background can be removed.

This proceeding will present the current status and the expected performance of the JUNO Top Tracker.
\end{abstract}

European Physical Society Conference on High Energy Physics - EPS-HEP2019 -

10-17 July, 2019

Ghent, Belgium

*Speaker. 


\section{The JUNO experiment}

The Jiangmen Underground Neutrino Observatory (JUNO) experiment is a multi-purpose neutrino experiment with the main objective of determining the neutrino mass hierarchy $(v \mathrm{MH})$ with a sensitivity better than $3 \sigma$ via reactor antineutrino oscillations [1]. It is located in the south of China at a distance of $53 \mathrm{~km}$ from the two major nuclear power plants providing most of the antineutrino $\left(\bar{v}_{e}\right)$ flux of JUNO. The JUNO detector consists of three main sub-detectors, the Central Detector (CD), the Water Cherenkov Detector (WCD), and the Top Tracker (TT). Fig. 1 shows the JUNO detector. Besides the $v \mathrm{MH}$, precise measurement of antineutrino spectra allows JUNO to be the first experiment to perform sub-percent precision measurement of the oscillation parameters $\theta_{12}$, $\Delta m_{21}^{2}$, and $\Delta m_{31}^{2}$ [1]. Thanks to the large detector volume and the good energy resolution, it will also contribute to non-reactor neutrino physics, including atmospheric, supernova, solar and geo neutrinos. The data taking of JUNO is currently planned to start in 2021.

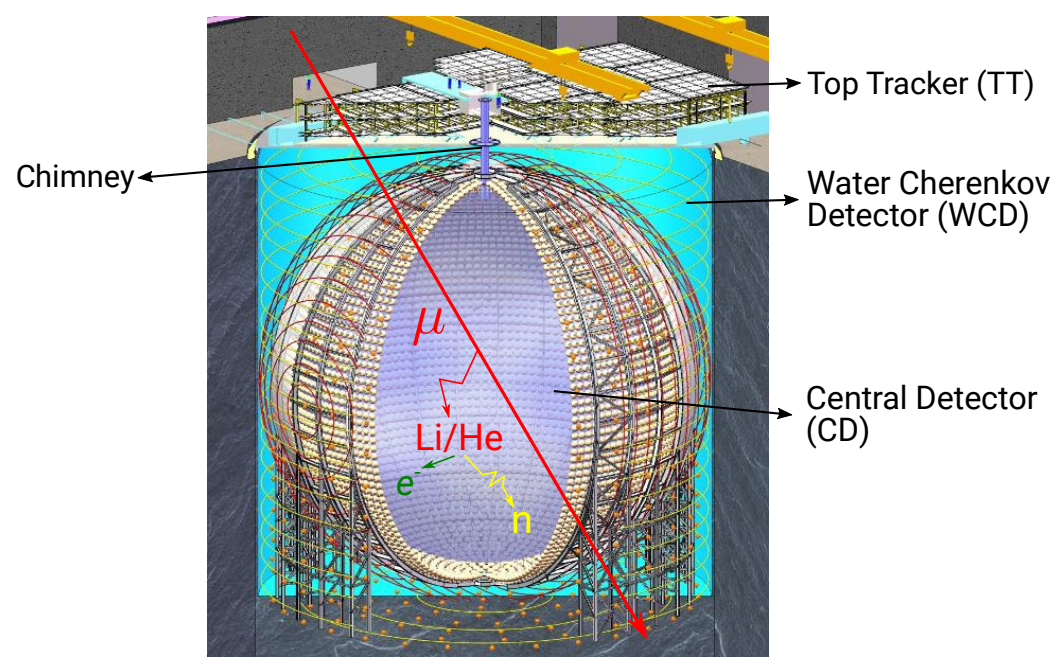

Figure 1: Schematic view of the JUNO detector. Cosmogenic isotope production is also illustrated, showing the parent muon, the induced isotopes and their decay products.

\section{Atmospheric muon veto}

The inverse beta decay (IBD) reaction is used in JUNO for reactor $\bar{v}_{e}$ detection. However, muons passing through the $\mathrm{CD}$ can produce radioisotopes, mainly ${ }^{9} \mathrm{Li}$ and ${ }^{8} \mathrm{He}$, which can mimic IBD events by decaying into an electron and a neutron, as shown in Fig. 1. Even thought the $700 \mathrm{~m}$ overburden lowers the muon rate in the $\mathrm{CD}$ to $3 \mathrm{~Hz}$, the expected cosmogenic isotope background has a rate similar to the IBD signal's before the muon veto [1], which was designed to remove specifically this background. It consists of establishing a cylindrical veto volume around the muon track with a radius of $3 \mathrm{~m}$ for a period of $1.2 \mathrm{~s}$. This period being a few times larger than the ${ }^{9} \mathrm{Li} /{ }^{8} \mathrm{He}$ half-lives, and the fact that the cosmogenic isotopes will be produced and stay close to their parent muon tracks, endow this strategy with a high veto efficiency, resulting in only 1.6 events per day remaining out of the initial 71 events per day. Since this veto strategy requires to know precisely where muon tracks cross the detector, its efficiency depends on the muon tracking precision. 


\section{Top Tracker}

The TT has the best muon tracking precision in JUNO, and it covers about $60 \%$ of the water pool top area, whereby it can detect $1 / 3$ of muons crossing the CD. It consists of $636.8 \times 6.8 \mathrm{~m}^{2}$ square walls made of plastic scintillator strips. The walls are arranged in a $3 \times 7$ grid in 3 layers. The wall in the middle of each layer is moved up in relation to the other layers to make space for the CD chimney, as shown in the left panel of Fig. 2. Each wall is composed of eight modules placed on two planes that are perpendicular to each other. One module contains 64 scintillator strips, which are read at both ends by two 64-channel multianode photomultiplier tubes (PMTs). The schematic view of a wall is shown in the right panel of Fig. 2. As these walls are taken directly from the OPERA experiment [2] Target Tracker without modification, the instrumental detail can be found in [3]. To adapt the walls for the TT, by contrast, new mechanical structure must be added and electronics is redesigned from the OPERA's.
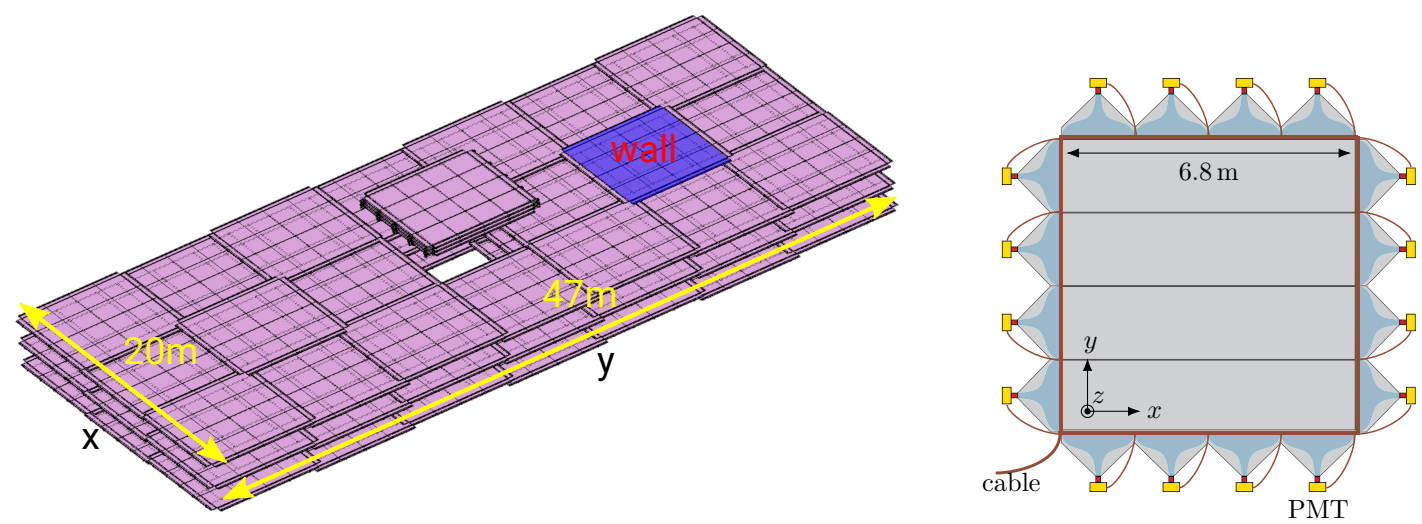

Figure 2: Left: schematic view of the Top Tracker. Right: schematic view of a Top Tracker wall.

This configuration allows the TT to detect muon hits with a spatial resolution of $2.6 \mathrm{~cm} \times$ $2.6 \mathrm{~cm} \times 1 \mathrm{~cm}$, and reconstruct tracks with a median angular resolution of $0.20^{\circ}$. In addition to being used directly as a veto detector, the TT tracking information can be used by other subdetectors to calibrate their own muon tracking algorithms. Lastly, the TT can detect some muons that cross neither the $\mathrm{CD}$ nor the WCD, but produce fast neutron background in the surrounding rock.

\section{Mechanical structure and ageing monitoring}

The walls were vertically suspended at OPERA, so the wall's mechanical strength was not a problem. However, since in JUNO they must be placed horizontally, a new supporting structure is required, and the TT will be mounted as shown in Fig. 3. The final design of this structure has been finished and approved by the collaboration. Tender to produce the structure is ongoing.

The walls had been fabricated over 13 years ago. An ageing monitoring has been started since 2015 to ensure that the scintillator performance is good. No noticeable ageing has yet been observed. 

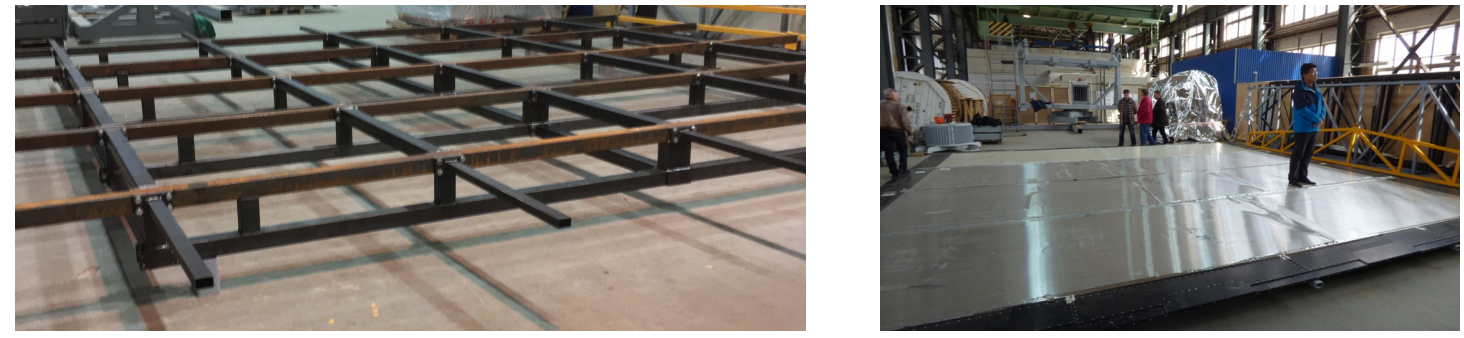

Figure 3: Left: the TT wall supporting structure. Right: a TT wall is mounted on the structure.

\section{Readout electronics}

The readout electronics consists of 1008 sets of Front-End Boards (FEBs) and Readout Boards (ROB), 63 Concentrator Boards (CBs), and one L2 trigger. The schematic design is depicted in the left panel of Fig. 4, while prototype versions of the first three cards are shown in the right panel of the same figure.

The PMT at the end of each TT module is attached directly to a FEB carrying a MAROC3 [4]. The MAROC3 provides trigger and analogue charge outputs for each channel. It can also perform the charge digitalisation through a built-in Wilkinson Analog-to-digital converter (ADC), which takes about $14 \mu$ s for one PMT hit. During this time, it will not digitalise any new hit, and thus the time can be regarded as its dead time. All ROBs on a wall transmit the trigger and charge information to the dedicated $\mathrm{CB}$. A ROB can also digitalise the FEB analogue charge output with its own Flash ADC. Moreover, it carries other functional units, such as slow control and high voltage.

The $\mathrm{CB}$ acts as an L1 trigger that makes trigger decision at wall level. If a PMT trigger is considered to be rejected, the $\mathrm{CB}$ will sent a signal to reset them, which makes it possible to reduce the dead time associated with the charge digitalisation of the FEB/ROB. To those accepted triggers, the $\mathrm{CB}$ will assign a timestamp with nanosecond precision. The CB will output valid L1 trigger signals to the L2 trigger, which further rejects events not induced by muons by looking at the global topology of the walls validated by the L1 in the whole TT. The trigger strategy of both trigger levels will be discussed later in Sec. 6 .

The FEB design was completed and the cards should be produced this year. The ROB design should be completed by the end of the year, with production foreseen in 2020. The CB design should be finished by 2020, and in particular the v2 of the CB is shown on the top right of Fig. 4. The L2 trigger is still in a conception stage.
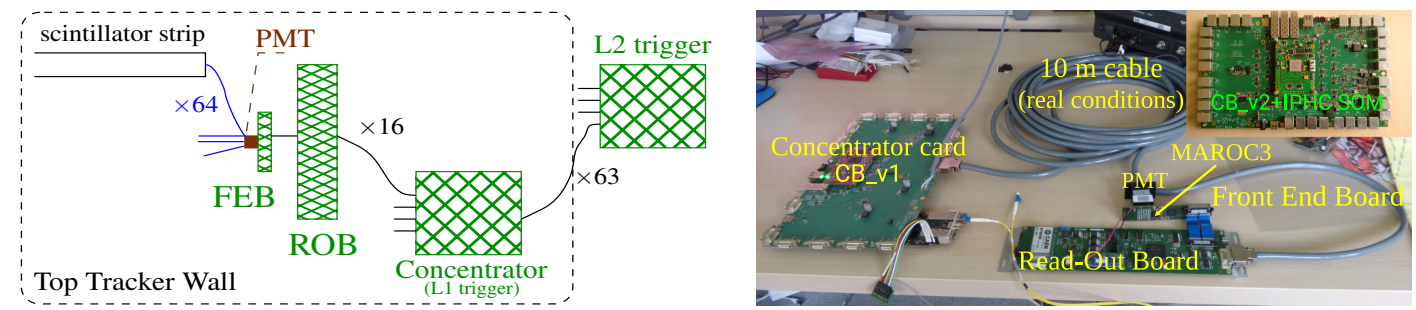

Figure 4: TT readout electronics schematic (left) and prototypes (right). 

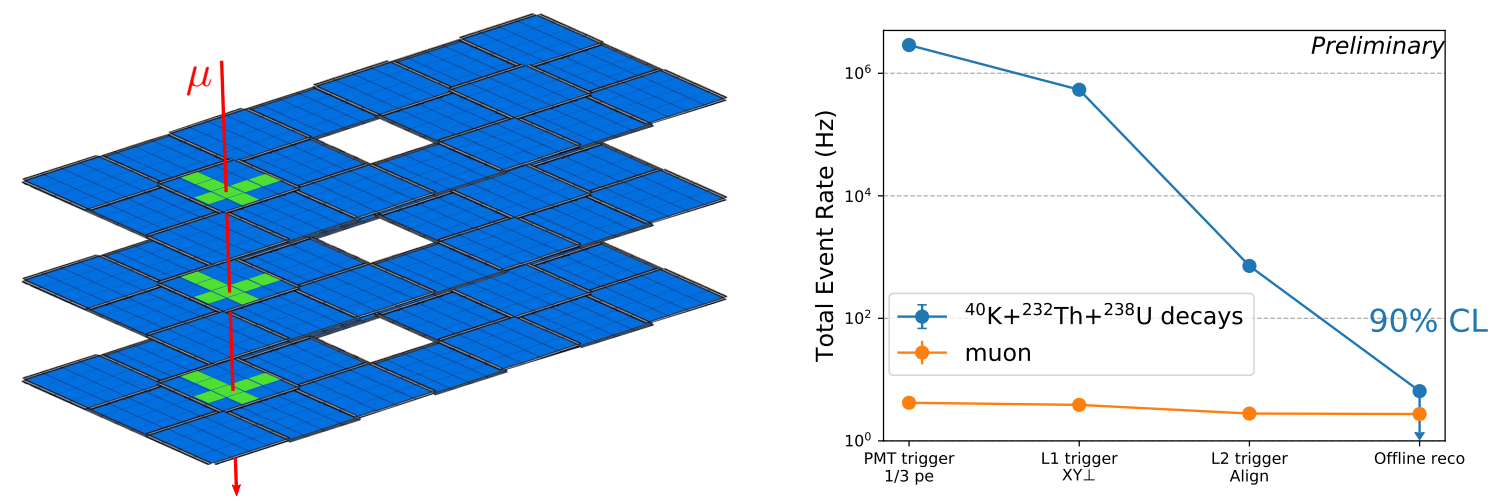

Figure 5: Left: a typical muon event detected by the TT. Right: event rates caused by radioactive decays (blue) and muons (orange) after each trigger level, and after the reconstruction.

\section{Trigger strategy}

The natural radioactivity level in the JUNO cavern is significantly higher than the one in Gran Sasso where OPERA was located. Each PMT trigger rate is estimated to be more than $50 \mathrm{kHz}$ mainly due to the decays of ${ }^{40} \mathrm{~K},{ }^{238} \mathrm{U}$, and ${ }^{232} \mathrm{Th}$. Given that the FEB dead time is about $14 \mu \mathrm{s}$, the system will be saturated without the L1 and L2 triggers. The trigger system was designed to identify muon hits on-the-fly, and reset the PMTs triggered by radioactive decays in order to reduce the dead time caused by charge readout. The left panel of Fig. 5 shows a typical muon event detected by the TT. Based on the topology of the triggered PMTs, the L1 trigger is designed to select PMT hits that form at least an XY coincidence, and the L2 trigger will accept only the aligned walls validated by the L1 trigger. However, the minimal is not the optimal. After studying different L1 trigger algorithms, the optimal one, for now, requires coincidences of 3 PMTs forming a T-shape $(\mathrm{XY} \perp)$. The reason to propose such an algorithm is that most muons can trigger PMTs at both ends of a TT module, whereas the radioactive decays rarely have the same capacity due to the smaller deposited energy, thus the rate from radioactivity is reduced.

The right panel of Fig. 5 exhibits the total TT event rates from radioactive decays and from muons with only the PMT trigger at 1/3 photoelectron (pe), and the corresponding rates after each trigger level, where the L1 trigger algorithm is $\mathrm{XY} \perp$. While the event rate of muons is barely decreased by the selections, the one of radioactive decays is significantly suppressed by 3 orders of magnitude after passing the L1 and L2 triggers, and thus can be handled by the system.

The last point of each curve represents the rate of successfully reconstructed muons. After the L1 and L2 triggers, no event from the simulated radioactive decays was reconstructed, so the last point shown in the curve corresponds to the upper limit at $90 \%$ confidence level, due to the limited simulation sample size used in this study. As a contrast, the reconstruction of muon events shows that the TT has a muon detection efficiency larger than $98 \%$ with these triggers.

\section{Acknowledgments}

This work has benefited from support by the initiative of excellence IDEX-Unistra (ANR-10IDEX-0002-02) from the French national programme "investment for the future". 


\section{References}

[1] F. An et al., Neutrino physics with JUNO, J. Phys. G 43 (2016) 030401 [1507 . 05613 ].

[2] R. Acquafredda et al., The OPERA experiment in the CERN to gran sasso neutrino beam, JINST 4 (2009) P04018.

[3] T. Adam et al., The OPERA experiment Target Tracker, Nucl. Instrum. Meth. A 577 (2007) 523 [physics.ins-det/0701153].

[4] S. Blin et al., MAROC, a generic photomultiplier readout chip, IEEE Nucl. Sci. Symp. Conf. Rec. 2010 (2010) 1690. 